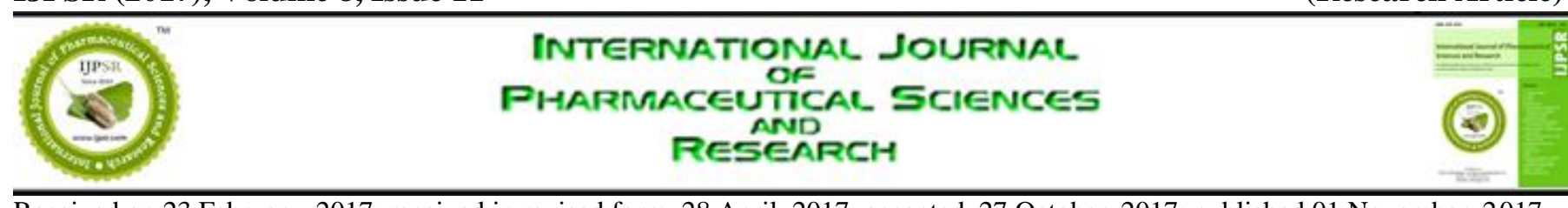

Received on 23 February, 2017; received in revised form, 28 April, 2017; accepted, 27 October, 2017; published 01 November, 2017

\title{
A PROSPECTIVE COMPARATIVE STUDY OF SAFETY OF LENALIDOMIDE PLUS DEXAMETHASONE COMBINATION THERAPY VERSUS VAD (VINCRISTINE, DOXORUBICIN AND DEXAMETHASONE) REGIMEN IN THE TREATMENT OF MULTIPLE MYELOMA
}

S. Remya ${ }^{1}$, M. J. Sudha ${ }^{* 1}$, R. Bindulatha Nair ${ }^{2}$ and K. L. Jayakumar ${ }^{3}$

Department of Pharmacology ${ }^{1}$, Azeezia Institute of Medical Sciences, Kollam - 691537, Kerala, India. Department of Pharmacology 2, Department of Radiotherapy ${ }^{3}$, Government Medical College, Thiruvanantapuram - 695011, Kerala, India.

\section{Keywords:}

Multiple myeloma, Lenalidomide plus Dexamethasone (Len-Dex) regimen, VAD (Vincristine,

Doxorubicin and Dexamethasone)

regimen, Safety profile, Adverse effects, Performance status.

Correspondence to Author:

Dr. M. J. Sudha

Associate Professor, Department of Pharmacology, Azeezia Institute of Medical Sciences, Kollam - 691537, Kerala, India.

E-mail: sudhasudhasudha@gmail.com

\begin{abstract}
Background: Lenalidomide plus Dexamethasone (Len-Dex) and VAD (Vincristine, Doxorubicin and Dexamethasone) regimen are the two common drug therapies involved in the treatment of Multiple myeloma. These two groups of drugs act by different mechanisms and their safety profile also varies. Objectives: To compare the safety of Len-Dex versus VAD regimen based on World Health Organization toxicity criteria by grade as well as the performance status of the patients of both the regimen by using Karnofsky performance status scale definitions rating. Materials and Methods: Eighty patients (forty in each arm) of newly diagnosed cases of multiple myeloma, who were willing to give the informed consent, were included in the study. Their baseline investigations and follow up investigations were collected at regular intervals, based on these values, the adverse effect profile as well as the performance status were evaluated and the results were compared and analyzed. Results: In Len-Dex regimen, constipation, leucopenia, thrombocytopenia, slow wound healing, sedation, renal toxicity and hepatotoxicity were high. VAD regimen produce higher incidence of nausea, vomiting, diarrhoea, anaemia and peripheral neuropathy. The study indicates that patients moved to higher scores with $14(35 \%)$ patients on VAD regimen and $17(42.5 \%)$ patients on Len-Dex achieving $90 \%$ with respect to the performance status. There was statistically significant $(p=0.023)$ performance status of patients after treatment with Len-Dex regimen. Conclusion: The tolerability as well as the overall performance status of patients of Lenalidomide-Dexamethasone (Len-Dex) combination therapy is clearly higher than that of VAD regimen among the study population.
\end{abstract}

INTRODUCTION: Multiple myeloma is one of the common plasma cell proliferative disorders and it is the second most common haematological malignancies. It is responsible for $15-20 \%$ of deaths from haematological malignancies and about $2 \%$ of all deaths from cancer ${ }^{1}$.

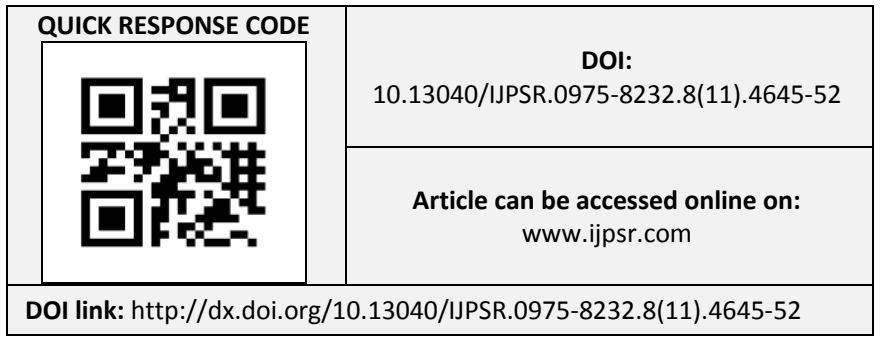

Plasma cell disorders cause neoplastic proliferation of the cells and the secretion of cell products like immunoglobulin molecules ${ }^{2}$. The use of anticancer agents like melphalan and corticosteroids paved a way to successful treatment of multiple myeloma ${ }^{3}$. Melphelan is a time tested orally effective alkylating agent which achieved remission by decreasing $\mathrm{M}$ protein, bone marrow plasma cells and Bence Jones proteinuria in a large number of patients 4,5 . Then a more effective combination therapy with Vincristine, Adriamycin and Dexamethasone (VAD) was introduced which has been very successful and is still continued. 
The VAD regimen was introduced for treatment of multiple myeloma in $1980 \mathrm{~s}^{6}$. The VAD is administered in a dose of vincristine $0.4 \mathrm{mg}$, doxorubicin $9 \mathrm{mg} / \mathrm{m}^{2}$ as continuous infusion every four hours for four days ${ }^{7}$. Dexamethasone is given orally as $40 \mathrm{mg}$ tablet on days $1-4,9-12$ and 17 20 in every 30 days ${ }^{8}$. The VAD regimen is used for inducing remission in patients who are newly diagnosed, relapsed or refractory to treatment 9 . The overall response rate was about $67 \%$. The related toxicities were neurotoxicity, fever, recurrent infections etc ${ }^{9}$.

Lenalidomide was developed as an alternative to thalidomide to improve its efficacy and reduce the toxicity ${ }^{10}$. Lenalidomide revimide, CC5013 is a synthetic glutamic acid derivative which is obtained from thalidomide. Lenalidomide is given along with dexamethasone as a combination therapy in the treatment of multiple myeloma ${ }^{11}$. The common adverse effects of lenalidomide include sedation, constipation, neuropathy, neutropenia and thrombocytopenia ${ }^{12}$. Lenalidomide leads to tumour flare reaction and tumor lysis syndrome, especially in patients with chronic lymphoid leukemia.

This is a fatal condition. To avoid this acute response, lenalidomide is gradually begun at a low dose. The low dose also reduces the chance of renal failure. The use of lenalidomide can rarely produce severe hepatic or renal toxicity. The combination therapy of lenalidomide with anthracyclin or glucocorticoids have a higher chance of thrombotic events. So usually along with these combinations, low dose heparin or other anticoagulants are added 13. The objective of this study was to evaluate the safety profile of these two commonly used regimens and to report the spectrum of adverse effects in patients on these two regimens.

MATERIALS AND METHODS: A prospective study was carried out in a tertiary care hospital after obtaining approval of the Institutional Review Board. The sample size was calculated using the data obtained from similar study.

The sample size was calculated using the following formula: ${ }^{14,2}$

$$
\mathrm{n}=\frac{2\left(\mathrm{Z} \alpha+\mathrm{Z}_{1}-\beta\right)^{2} \mathrm{pq}}{\mathrm{d}^{2}}
$$

Where ' $n$ ' is the sample size

' $p$ ' is calculated from similar study from literature,

$$
\text { i.e. } \frac{p^{1}+p^{2}}{2}
$$

$p^{1}=$ efficacy of Len-Dex (lenalidomide dexamethasone) regimen in multiple myeloma i.e. about $91 \%$

$p^{2}=$ efficacy of $\mathrm{VAD}$ regimen in multiple myeloma i.e. about $63 \%$

$$
\begin{aligned}
& q=100-p \\
& d=p^{1}-p^{2}
\end{aligned}
$$

At 5\% significance level, $\mathrm{Z} \alpha$ is 1.96

$$
\begin{aligned}
& \text { At } 80 \% \text { power, } \mathrm{Z}_{1-\beta} \text { is } 0.842 \\
& \mathrm{n}=\frac{2 \times(7.85) \times 77 \times 23}{(91-63.7)^{2}} \approx 38
\end{aligned}
$$

So the minimum sample size required in each treatment arm was fixed as 40 .

Institutional ethics committee approval is taken before the study. At the time of enrolling patients into the study, a detailed written informed consent was taken from each participant.

The study included patients of both sex with newly diagnosed multiple myeloma with the following clinical features like, Patients with bone marrow plasma cells $20 \%$ or more, Patients with measurable disease defined as serum monoclonal protein level $>10 \mathrm{~g} / \mathrm{L}$, Patient with lytic bone lesions., Patients with ' $\mathrm{M}$ ' band on electrophoresis., Patients with $\mathrm{Hb}>8 \mathrm{mg} / \mathrm{L}$, Patients with platelet count $>100 \times 10^{3} / \mathrm{L}$, Patients with absolute neutrophil count $>1.5 \times 10^{3} / \mathrm{L}$ and Patients with urine creatinine level $<2.5 \mathrm{mg} / \mathrm{dL}$.

Severely ill patients, patients with deep vein thrombosis, patients with uncontrolled infections and patients with other co - existing malignancies are excluded from the study. The study was started only after obtaining written informed consent from the patients. Information regarding patients' demographics, family history, education and occupation were obtained by asking leading questions and was recorded in the proforma. On the first visit, a detailed history was taken and clinical examination was performed before initiation of treatment. Baseline investigation reports like haemoglobin, TC, DC, ESR, platelet count, bleeding time, 
clotting time, serum levels of calcium, phosphorus, and $\mathrm{M}$ protein, LFT, RFT, ECG, X-ray skull and bone marrow examination were recorded in the proforma. Patients were allocated by the treating physician and one group was given Len-Dex (lenalidomide + dexamethasone) regimen and the other VAD (Vincristine, Adriamycin, Dexamethasone) regimen. A total of six cycles were given for both groups. The dosing schedule of each cycle is as follows:

Patients put on Len-Dex regimen was administered lenalidomide in the dose of $25 \mathrm{mg}$ orally four times daily from day $1-21$. The same patients received dexamethasone $40 \mathrm{mg}$ orally daily on days $1,8,15$, 22 of chemotherapy ${ }^{15}$. Patients on VAD regimen received vincristine in the dose of $0.4 \mathrm{mg}$ iv bolus and doxorubicin $9 \mathrm{mg} / \mathrm{m}^{2}$ iv infusion over 2 hour, daily from day 1 - 4 and dexamethasone in the dose of $40 \mathrm{mg}$ orally daily on days $1-4,9-12$ and 17 20. There was an interval of four weeks in between the cycles of both regimens.

The patients reported to the physician before starting each cycle with all the baseline investigations repeated except bone marrow study, which was done only before and after completion of the treatment. All data were entered in the proforma before treatment, after each cycle and after completion of treatment which include:

- Detailed history including that of any adverse effects

- Detailed clinical examination

- Laboratory investigation reports

TABLE 1: WHO CRITERIA FOR GRADING OF TOXICITY

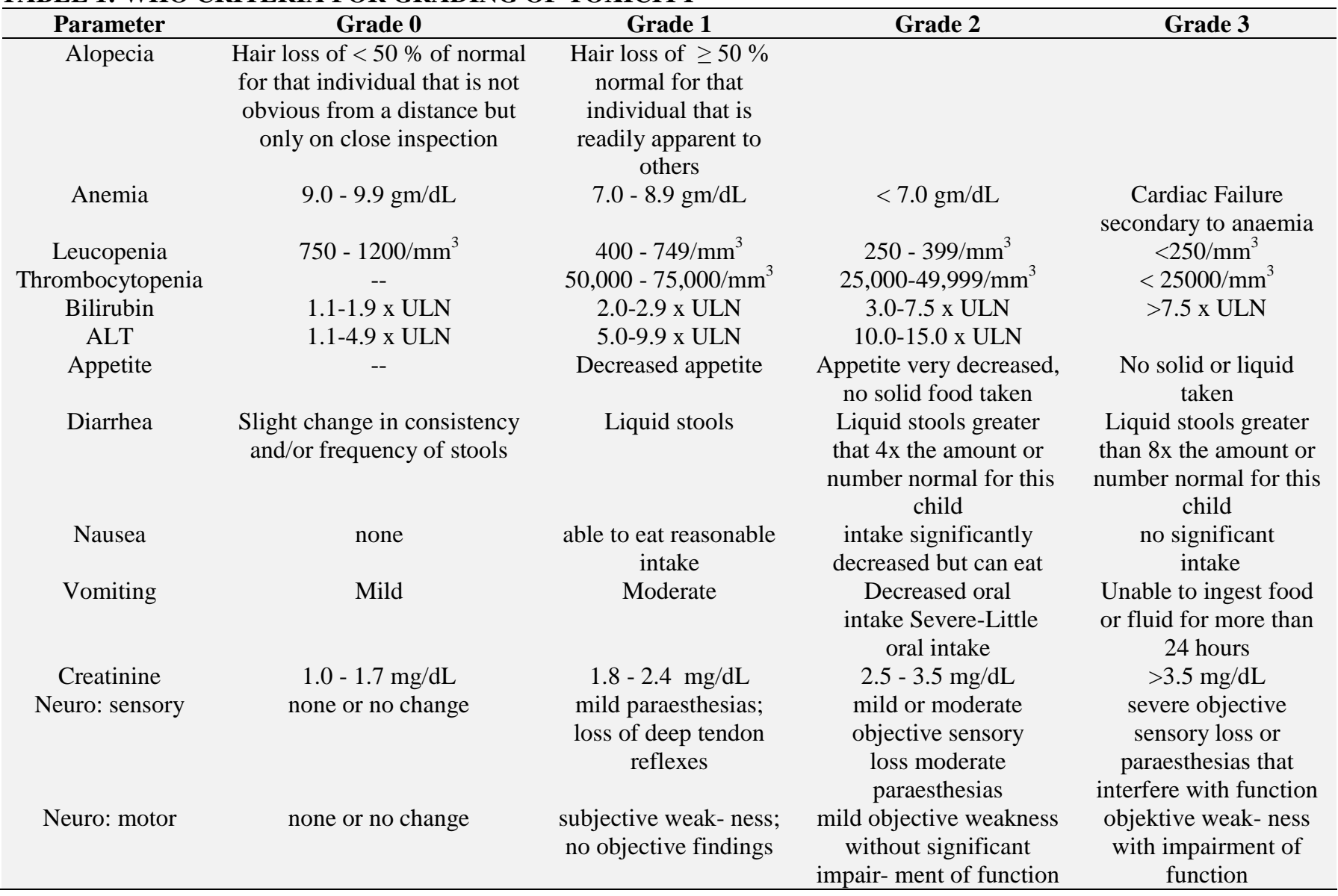

The analysis of adverse effects of both these regimen were evaluated based on the filled proforma at the end of the study. The analysis was done based on WHO (World Health Organization) toxicity criteria by grade. The performance status of the patients of both the regimen were evaluated using Karnofsky performance status scale definitions rating $(\%)$ criteria.

\section{RESULTS:}

Demographic Profile: The age range of the study population was between 40 and 80 years. The mean 
age of total patients is 62.8 years, while that of $\mathrm{VAD}$ regimen is 64.3 years and Len-Dex regime is 61.2 years. Out of the total of 80 patients, $48(60 \%)$ were males and $32(40 \%)$ were females. Among patients put on VAD regimen $27(67.5 \%)$ were males and $13(32.5 \%)$ were females. In Len-Dex regimen group, there were 21 males $(52.5 \%)$ and 19 females $(47.5 \%)$.

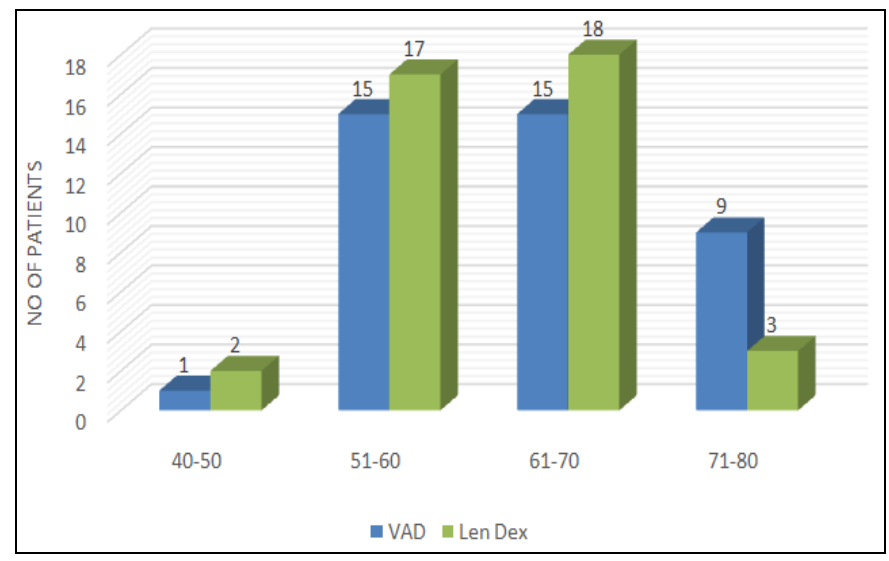

FIG. 1: AGE WISE DISTRIBUTION OF PATIENTS ON VAD AND LEN-DEX REGIMENS

Clinical Presentation and Adverse Effects Profile (Table 2): The patients presented with different symptoms, fatigue being the most common and shown by all patients.

TABLE 2: CLINICAL PRESENTATION OF MULTIPLE MYELOMA

\begin{tabular}{cc}
\hline Clinical feature & \% of patients \\
\hline Fatigue & 100 \\
Loss of weight & 97.5 \\
Loss of appetite & 96.3 \\
Pallor & 95 \\
Bone pain & 91.3 \\
Frequent infections & 91.3 \\
Insomnia & 78.8 \\
Fever & 57.5 \\
Fracture & 41.3 \\
Palpitation & 21.3 \\
Swelling of legs & 12.5 \\
\hline
\end{tabular}

Overall Comparison of Toxicities: Toxicities like nausea, vomiting, diarrhoea, anemia, and peripheral neuropathy are more in patients administered VAD regimen, whereas constipation, leucopenia, thrombocytopenia, elevated levels of serum creatinine, bilirubin and liver enzymes were higher in patients on Len-Dex regimen (Table 3).

Dermatological Toxicity: Alopecia is a very common adverse effect with most of the anticancer agents. Table 4 shows that majority of patients developed grade 1 alopecia, $33(82.5 \%)$ patients on VAD regimen and $32(80 \%)$ on Len-Dex regimen.

Gastrointestinal adverse effects (Table 4): Anorexia was seen in all patients in both regimes. Nausea was present in all patients who received VAD regimen but was absent in patients on LenDex regimen. Some patients complained of constipation in one visit and diarrhoea in a different visit and vice versa. Only one patient on VAD regimen was free of vomiting. 20 patients on VAD regimens and 30 on Len - Dex developed constipation. Though diarrhea was reported in both arms - none from Len-Dex regimen went into grade 3 diarrhoea.

\begin{tabular}{ccc} 
TABLE 3: OVERALL COMPARISON & OF TOXICITIES \\
\hline Toxicity & $\begin{array}{c}\text { VAD regimen } \\
\text { (N=40) }\end{array}$ & $\begin{array}{c}\text { Len-Dex } \\
\text { regimen }(\mathbf{N}=\mathbf{4 0})\end{array}$ \\
\hline Alopecia & 39 & 39 \\
Nausea & 40 & 0 \\
Vomiting & 39 & 0 \\
Constipation & 20 & 30 \\
Diarrhoea & 23 & 14 \\
Anemia & 38 & 24 \\
Leucopenia & 21 & 32 \\
Thrombocytopenia & 14 & 16 \\
Elevated serum & 14 & 18 \\
creatinine levels & & \\
Hyperbilirubinemia & 10 & 15 \\
Elevated liver enzymes & 30 & 33 \\
Peripheral neuropathy & 28 & 27 \\
\hline
\end{tabular}

TABLE 4: GASTROINTESTINAL ADVERSE EFFECTS - COMPARISON OF VAD AND LEN-DEX REGIMENS

\begin{tabular}{ccc}
\hline Grade & VAD regimen (N=40) & Len-Dex regimen $(\mathbf{N}=\mathbf{4 0})$ \\
\hline \multicolumn{3}{c}{ Nausea } \\
\hline 0 & $0(0 \%)$ & $40(100 \%)$ \\
1 & $26(65 \%)$ & $0(0 \%)$ \\
2 & $14(35 \%)$ & $0(0 \%)$ \\
\hline \multicolumn{3}{c}{ Vomiting } \\
\hline 0 & $1(2.5 \%)$ & $40(100 \%)$ \\
1 & $3(7.5 \%)$ & $0(0 \%)$ \\
2 & $31(77.5 \%)$ & $0(0 \%)$ \\
3 & $5(12.5 \%)$ & $0(0 \%)$ \\
\hline \multicolumn{3}{c}{ Constipation } \\
\hline 0 & $20(50 \%)$ & $10(25 \%)$ \\
1 & $1(2.5 \%)$ & $24(60 \%)$ \\
2 & $19(47.5 \%)$ & $6(15 \%)$ \\
\hline \multicolumn{3}{c}{ Diarrhea } \\
\hline 0 & $17(42.5 \%)$ & $26(65 \%)$ \\
1 & $1(2.5 \%)$ & $11(27.5 \%)$ \\
2 & $21(52.5 \%)$ & $3(7.5 \%)$ \\
3 & $1(2.5 \%)$ & $0(0 \%)$ \\
\hline
\end{tabular}

Hematological toxicities: It is shown in Table 5, Grade 2 anemia is seen in $33(82.5 \%)$ and $22(55 \%)$ 
of patients in VAD and Len-Dex regimens respectively. Very few patients in both regimens went into grade 3 with $5(12.5 \%)$ on VAD regimen and $2(5 \%)$ on Len-Dex regimen.

Grade 1 leucopenia was seen in significantly more $(52.5 \%)$ patients on VAD regimen than in patients on Len-Dex regimen (80 \%). None of the patients in either regimens developed higher grades of leucopenia. A total of 30 patients developed thrombocytopenia of grade 1 with 14 patients (35\%) on VAD regimen and 16 patients $(40 \%)$ on Len-Dex regimen. None of them went into higher grades. The difference was not statistically significant $\left(\chi^{2}=0.213, d f=1, p=0.644\right)$.

Renal Adverse Effects (Fig. 2): Grade 1 elevation was seen in $14(35 \%)$ patients on VAD regimen and $18(45 \%)$ of them on Len-Dex regimen. Serum creatinine values did not increase to higher levels of grade 2 or more in any of the patients on either regimen.

Hepatic Adverse Effects (Table 6): Len-Dex patients had statistically more hyperbilirubinemia than those who were on VAD. There was no significant difference in serum alanine aminotransferase.

Neurological Toxicity: Sensory type of peripheral neuropathy with mild parasthesia and loss of deep tendon reflexes (grade 1) was seen in $28(70 \%)$ patients on VAD regimen and $27(67.5 \%)$ on Len Dex regimen. $12(30 \%)$ on VAD regimen and 13 $(32.5 \%)$ on Len-Dex did not show any sensory changes. This difference is not statistically significant $(\mathrm{p}=0.81)$.

TABLE 5: ANEMIA - COMPARISON OF VAD AND LEN-DEX REGIMENS, $* \mathbf{P}<0.05$

\begin{tabular}{ccc}
\hline Grade & VAD regimen $(\mathbf{N}=\mathbf{4 0})$ & Len-Dex regimen $(\mathbf{N}=\mathbf{4 0})$ \\
\hline 1 & Anaemia & $16(40 \%)$ \\
2 & $2(5 \%)$ & $22(55 \%)$ \\
3 & $33(82.5 \%)$ & $2(5 \%)$ \\
& $5(12.5 \%)$ & \\
0 & Leucopenia & $8(20 \%)$ \\
1 & $19(47.5 \%)$ & $32(80 \%)^{*}$ \\
& $21(52.5 \%)$ & \\
0 & Thrombocytopenia & $24(60 \%)$ \\
1 & $26(65 \%)$ & $16(40 \%)$ \\
\hline
\end{tabular}

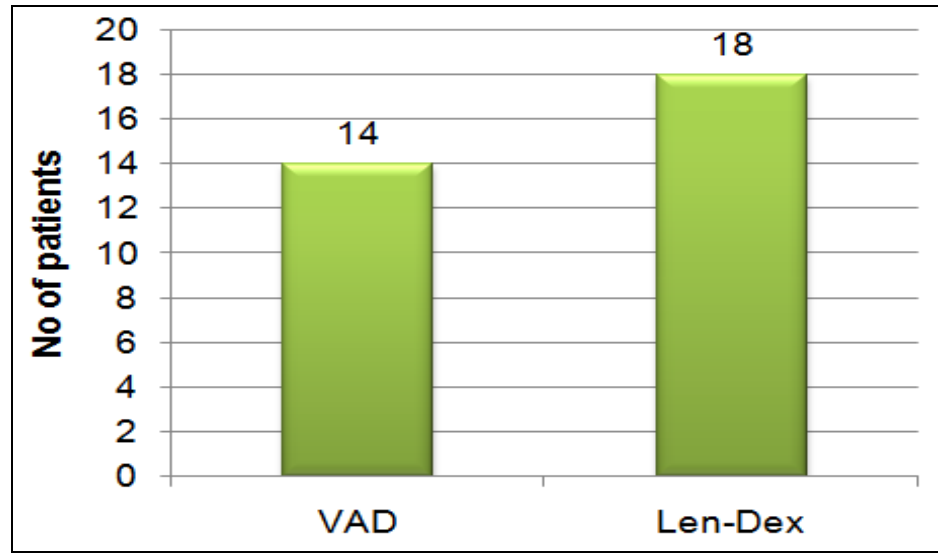

FIG. 2: ELEVATED SERUM CREATININE - COMPARISON OF VAD AND LEN-DEX REGIMENS

TABLE 6: COMPARISON OF VAD AND LEN-DEX REGIMENS

\begin{tabular}{ccc}
\hline Grade & VAD regimen $(\mathbf{N}=\mathbf{4 0})$ & Len-Dex regimen $(\mathbf{N}=\mathbf{4 0})$ \\
\hline 0 & $30(75 \%)$ & $25(62.5 \%)$ \\
1 & $10(25 \%)$ & $15(37.5 \%)$ \\
\hline \multicolumn{3}{c}{ Serum alanine aminotransferase } \\
\hline 1 & $10(25 \%)$ & $7(17.5 \%)$ \\
2 & $25(62.5 \%)$ & $25(62.5 \%)$ \\
\hline
\end{tabular}




\section{Other Toxicities:}

TABLE 7: OTHER TOXICITIES- COMPARISON OF VAD AND LEN-DEX REGIMENS

\begin{tabular}{ccccc}
\hline & VAD regimen $(\mathbf{N}=\mathbf{4 0})$ & Len-Dex regimen $(\mathbf{N}=\mathbf{4 0})$ & $\chi^{2}$ & P value \\
\hline Upper respiratory infections & $40(100 \%)$ & $40(100 \%)$ & - & - \\
Loss of weight & $40(100 \%)$ & $40(100 \%)$ & - & - \\
Slow wound healing & $32(80 \%)$ & $40(100 \%)$ & 8.889 & 0.003 \\
Fever & $31(77.5 \%)$ & $33(82.5 \%)$ & 6.31 & 0.576 \\
Pedal edema & $28(70 \%)$ & $27(67.5 \%)$ & 0.06 & 0.819 \\
Dyspnoea & $23(57.5 \%)$ & $28(70 \%)$ & 1.35 & 0.244 \\
Palpitation & $13(32.5 \%)$ & $15(37.5 \%)$ & 0.22 & 0.639 \\
Sedation & $8(20 \%)$ & $12(30 \%)$ & 1.07 & 0.301 \\
\hline
\end{tabular}

There was no mortality in either regimen during the study period.

Performance Status of Patients: Performance status of patients is an indicator of quality of life of the patient and his ability to survive chemotherapy. It was assessed by using Karnofsky scoring scales which runs from 100 - 0 with $100 \%$ indicating perfect health and no complaints, and $0 \%$ denoting death. In this study assessment of the performance status before treatment was distributed between 60 and $80 \%$ in most of the patients in both the regimens. Fig. 3 indicates that patients moved to higher scores with $14(35 \%)$ patients on VAD regimen and $17(42.5 \%)$ patients on Len-Dex achieving $90 \%$. Only a few numbers of patients were in lower grade of $60 \%$. Analysis was done using chi square test $\left(\chi^{2}=5.165, d f=1, p=0.023\right)$ which shows a statistically significant performance status of patients after treatment with Len-Dex regimen.

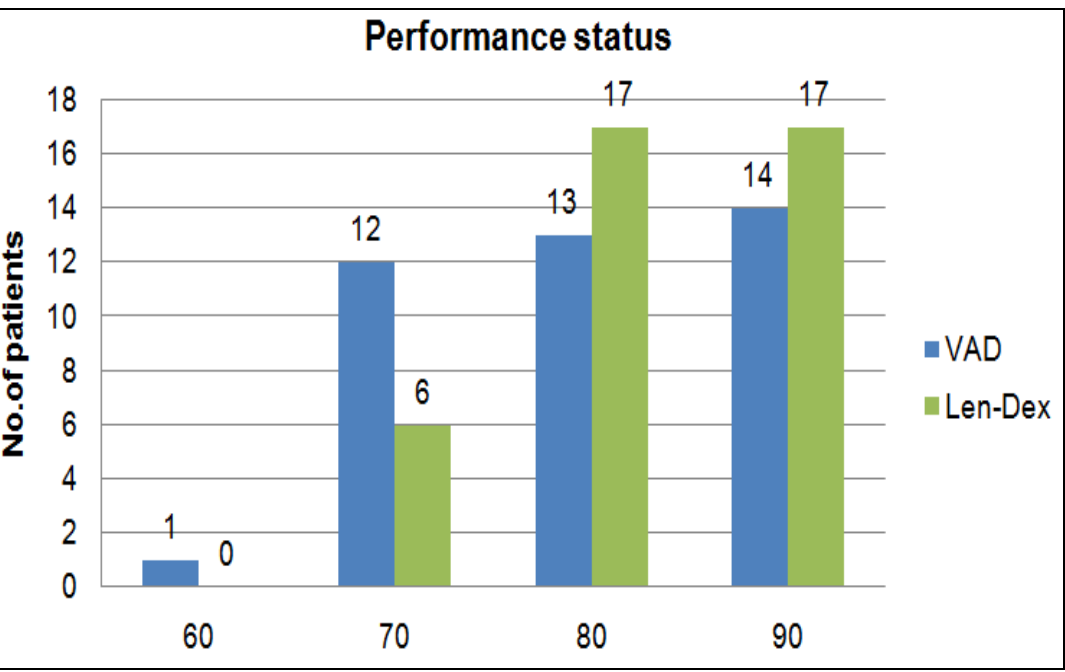

FIG. 3: COMPARISON OF PERFORMANCE STATUS OF PATIENTS AFTER TREATMENT

DISCUSSION: The safety of the regimens was measured by assessing the development of various adverse effects and grading them according to the WHO toxicity criteria. It was found that alopecia was found with both regimens but majority of them developed grade 1 alopecia. A few of them also went into grade 2 in both regimens. This shows that there is no significant difference between the two regimens in terms of alopecia. Nausea was not seen in any of the patients on Len-Dex regimen but all of them on VAD regimen developed nausea and some of them also went into grade 2 . In the study done by Rajkumar SV et al., ${ }^{11}$, nausea was seen in $6 \%$ of patients. A similar profile was also seen in the study done by Falco et al., ${ }^{16}$.Vomiting was also absent in all patients who received Len-Dex regimen in our study which is consistent with other studies. Majority of patients on VAD regimen developed vomiting; some of them going into grade 3. Constipation was a predominant adverse effect in patients receiving both the regimens. $50 \%$ patients on VAD and $75 \%$ patients on Len-Dex had complaints of constipation. 
However grade 2 constipation was seen in lesser number of patients on Len-Dex regimen. Other studies done by Rajkumar SV et al., ${ }^{11}$ and Falco et $a l^{16}$ also have reported incidence of constipation with Len-Dex regimen. The incidence of constipation in Len-Dex regimen was about $27 \%$ in the study conducted by Dimopoulos MA et al., ${ }^{17}$. Constipation was reported in patients on VAD regimen in study by Zhongguo Shi Yan Xue Ye Xue Za Zhi ${ }^{18}$. Diarrhoea was also reported in patients on both the regimens. $35 \%$ patients on Len-Dex regimen and $57.5 \%$ patients on VAD regimen had diarrhoea.

This is consistent with studies done by Rajkumar SV et al., ${ }^{11}$. In the study conducted by Dimopoulos MA et al., ${ }^{17}$ showed that incidence of diarrhoea in Len-Dex regimen was up to $50 \%$.

Hematological toxicities are consistent with the studies done by by Rajkumar SV et al., ${ }^{11}$, Zonder JA et al. ${ }^{19}$ and Dimopoulos MA et al., ${ }^{17}$ on LenDex regimen. However the incidences of all haematological toxicities were higher in our study population with both regimens. Comparison of nephrotoxicity based on serum creatinine shows that both regimens caused the increase in serum creatinine levels with a higher incidence in patients on Len-Dex regimen (45\%) as compared to VAD regimen $(35 \%)$. Elevated liver enzyme was also more with Len-Dex regimen but the difference is only marginal.

In the study by Rajkumar SV et al., ${ }^{11}$ showed that the incidence of hyperbilirubinemia and elevated liver enzymes in Len-Dex regimen was $6 \%$ and 12 $\%$ respectively. In the study conducted by Segeren $\mathrm{CM}$ et al., showed that the incidence of neurotoxicity with VAD regimen is about $22 \% ?$. Peripheral neuropathy was a common adverse effect of VAD regimen observed by study of Taleb FA et al., also ${ }^{20}$. Other toxicities like frequent upper respiratory infections, loss of weight and slow wound healing were present in almost all patients of both regimens. Fever, dysponea, pedal edema, palpitation and sedation were the other mild toxicities observed in the study. All these toxicities were mild in nature in both the treatment groups.

In a study done by Dimopoulos $\mathrm{M}^{17}$ showed that these toxicities were common in Len-Dex regimen which is consistent with our study. Similar results are also seen in the study by Weber DM ${ }^{21}$. Fever is a common presentation in VAD regimen as stated by Mashhad Ali M $^{22}$.

The performance status of the patients is an indicator of quality of life which also points to the efficacy of treatment regimen. The performance status as measured using Karnofsky scale shows that more patients moved to higher scale after treatment with both regimens. However it was found that all patients on Len-Dex regimen had a scale above $70 \%$ with majority of them (34 patients) achieving more than $80 \%$.

CONCLUSION: Adverse effect profile shows that some adverse effects like nausea, vomiting are significantly less with Len-Dex regimen whereas others like constipation, leucopenia, thrombocytopenia, slow wound healing, sedation, renal toxicity and hepatotoxicity are high. VAD regimen produce higher incidence of nausea, vomiting, diarrhoea, anemia and peripheral neuropathy. Both regimens showed almost equal incidence of frequent upper respiratory infections, loss of weight, fever, pedal oedema, palpitations etc. The better tolerability of Len-Dex regimen offers a better quality of life to the patients. Long term study should be undertaken to assess disease progression and longevity. The performance status of the study population accessed using Karnofsky performance score evaluation showed that the overall improvement of performance status was higher in Len-Dex regimen compared to VAD regimen

ACKNOWLEDGEMENT: Authors thank all patients who took part in the study.

CONFLICT OF INTEREST: Authors declare that there is no conflict of interest

\section{REFERENCES:}

1. Smith: Multiple myeloma. BMJ 2013; 346:f3863

2. Munshi NC, Longo DL, Anderson KC, et al., Plasma cell disorders. In: Dan L Longo, Dennnis L Klasper, J Jarry Jameson et al., (eds.) Harrisons Principles of Internal Medicine. New York: Mc Grew Hill Medical; Edition18, 2012; 936-942.

3. Kyle RA: Multiple myeloma: an odyssey of discovery. British Journal of Hematology 2000; 111(4):1035-1044.

4. Hogan MC, Lee A, Soleberg LA and Thome SD: Unusual presentation of multiple myeloma with unilateral visual 
loss and numb chin syndrome in a young adult. American Journal of Hematology 2002; 70(1); 55-59.

5. Takiar R, Nadayil D and Nandakumar A: Projections of number of cancer cases in India (2010-2020) by cancer groups. Asian Pac J Cancer Prev 2010; 11(4): 1045-1049.

6. Kyle RA and Rajkumar SV: Multiple myeloma. Blood 2008; 111: 2962-2972.

7. Rajkumar SV: Multiple myeloma: the death of VAD as initial therapy. BLOOD, Journal of American Society of Hematology; 2005; 106: 2-3.

8. Stewart K: The American School of Oncology-Multiple Myeloma: A Practical Guide to Current Management, Atlanta: Network for Medical Communication and Research, LLC 2005.

9. Segeren CM, Sonnveld P, Holt BVD et al., Vincristine, Doxorubicin and Dexamethasone (VAD) administered as rapid intravenous infusion for first-line treatment in untreated multiple myeloma. British Journal of Hematology 1999; 105(1): 127-130.

10. Thomas SK, Richards TA and Weber DM: Lenalidomide in multiple myeloma; Best Practise and Research Clinical Haematology 2007; 20(4): 717-735.

11. Rajkumar SV, Hayman SR, Lacy MQ, Dispenzieri A, et al., Combination therapy with lenalidomide plus dexamethasone (REV/DEX) for newly diagnosed myeloma. Blood 2005; 106(13): 4050-4053.

12. Sviggum HP, Davis MDP, Rajkumar SV and Dispenzieri A: Dermatologic adverse effects of lenalidomide therapy for amyloidosis and multiple myeloma. JAMA Dermatology, Arch Dermatol 2012; 142 (10): 1298-1302.

13. Bruce A Chabner, Jeffrey Barner, James Cleary et al., Pharmacology and toxicity of antineoplastic drugs. In: Kenneth Kaushansky, Ernest Beutles, Marshall A Lichtman et al., (eds.) Williams Hematology. Edition 8, New York: Mc Grew Hill Medical; 2010: 283-312.
14. Friese CR, Abel GA, Magazu LS, et al., Diagnostic delay and complications for older adults with multiple myeloma, Leukemia Lymphoma 2009; 50(3): 392-400.

15. Dispenzieri A, Lacy MQ, Greipp PR: Multiple myeloma. In: Greer JP, Foerster J, Rodgers GM et al., (eds.) Wintrobes Clinical Hematology. Philadelphia: Wolter Klauwer/Lippincott Williams and Wilkins; Edition 12, 2009; 2372-2420.

16. Patrizia F, Liberati LFA, Marina/A Musto, Pellegrino, et $a l$., Lenalidomide and its role in the management of multiple myeloma; Expert Rev Anticancer Ther. 2008; 8(6): 865-74.

17. Dimopoulos MA, Kastritis E and Rajkumar SV: Treatment of plasma cell dyscrasias with lenalidomide, Leukemia 2008; 22: 1343-1353.

18. Shi Z, Xue Y, Xue Y and Zhi Z: Therapeutic efficacy analysis VD regimen and VAD regimen for multiple myeloma; 2013: 21(3): 647-9.

19. Zonder JA, Crowley J, Hussein MA, et al., Lenalidomide and high-dose dexamethasone compared with dexamethasone as initial therapy for multiple myeloma: a randomized Southwest Oncology Group trial (S0232). Blood 2010; 116(26): 5838-41.

20. Taleb FA, Far AE, El-Sawy W, et al., VAD regimen as front line therapy in multiple myeloma; Journal Of The Egyptian National Cancer Institute 2001; 13(4); 245-250.

21. Weber DM, Chen C and Niesvizky R: Lenalidomide plus Dexamethasone for relapsed multiple myeloma in North America. N Engl J Med 2007; 357: 2133-2142.

22. Mashhad AM, Mehdi $H$ and Negin K: VAD regimen as initial treatment for multiple myeloma, International Journal of Hematology-Oncology and Stem Cell Research (IJHOSCR). 2010; 4(4): 22-24.

How to cite this article:

Remya S, Sudha MJ, Nair RB and Jayakumar KL: A prospective comparative study of safety of lenalidomide plus dexamethasone combination therapy versus VAD (Vincristine, Doxorubicin and Dexamethasone) regimen in the treatment of multiple myeloma. Int $\mathrm{J}$ Pharm Sci Res 2017; 8(11): 4645-52.doi: 10.13040/IJPSR.0975-8232.8(11).4645-52.

All @ 2013 are reserved by International Journal of Pharmaceutical Sciences and Research. This Journal licensed under a Creative Commons Attribution-NonCommercial-ShareAlike 3.0 Unported License.

This article can be downloaded to ANDROID OS based mobile. Scan QR Code using Code/Bar Scanner from your mobile. (Scanners are available on Google Playstore) 